\title{
Origin and possible birthplace of the extreme runaway star HIP 60350
}

\author{
P. Tenjes ${ }^{1,2}$, J. Einasto ${ }^{2}$, H. M. Maitzen ${ }^{3}$, and H. Zinnecker ${ }^{4}$ \\ 1 Institute of Theoretical Physics, Tartu University, Tähe 4, Tartu 51010, Estonia \\ 2 Tartu Observatory, 61602 Tõravere, Estonia \\ 3 Institut für Astronomie der Universität Wien, Türkenschanzstrasse 17, 1180 Wien, Austria \\ 4 Astrophysikalisches Institut Potsdam, An der Sternwarte 16, 14482 Potsdam, Germany
}

Received 28 September 2000 / Accepted 15 December 2000

\begin{abstract}
Using the recently determined spatial velocity components of the extreme runaway star HIP 60350 and a gravitation potential model of the Galaxy, we integrate the orbit of HIP 60350 back to the plane of the Galaxy. In this way, a possible location of the formation of the star is determined. We estimate the uncertainty of the result due to the uncertainties of the gravitational potential model and the errors in the spatial velocity components. The place of birth lies (within the errors) near the position of the open cluster NGC 3603. However, the ejection event which occurred about $20 \mathrm{Myr}$ ago is in contradiction with the cluster mean age of $3-4 \mathrm{Myr}$. We suggest that it occurred at an earlier phase in sequential star formation in that region. We discuss also ejection mechanisms. Due to the rather high mass of the star (about $5 M_{\odot}$ ), the most probable model is that of dynamical ejection.
\end{abstract}

Key words. stars: formation - stars: kinematics - stars: individual HIP 60350 - open clusters and associations: NGC 3603

\section{Introduction}

Recent Hipparcos proper motion measurements for the star HIP 60350, together with its radial velocity, allowed the determination of its spatial velocity components $U=$ $+352 \mathrm{~km} \mathrm{~s}^{-1}, V=+183 \mathrm{~km} \mathrm{~s}^{-1}$ and $W=+130 \mathrm{~km} \mathrm{~s}^{-1}$, giving for the total velocity the value $v=417 \mathrm{~km} \mathrm{~s}^{-1}$ (Maitzen et al. 1998). These results suggest the star is a rare extreme runaway star. The origin of such a high velocity is not clear, especially given the rather high mass of the star, $M \simeq 5 M_{\odot}$. Thus, we have chosen to study the possible origin of the velocity: first the location of birth, and then the mechanisms allowing the acquisition of such a high velocity. Maitzen et al. (1998) calculated the distance of HIP 60350 from the Sun, about $3.5 \mathrm{kpc}$. Together with its equatorial coordinates, this gives us galactocentric cylindrical coordinates of HIP $60350, R=9.25 \mathrm{kpc}$, $\theta=3.3^{\circ}, z=3.4 \mathrm{kpc}$ (zero point of the azimuthal angle is in the direction to the Sun). Hence, both the velocities and the coordinates of the star are known.

The star HIP 60350 is of spectral type B4-5V. The high velocity carries it far from the galactic plane, towards the outer halo. For this reason, the study of its orbit is interesting in relation to another problem, namely the origin of young stars observed in galactic halos. Recent surveys of

Send offprint requests to: P. Tenjes, e-mail: tenjes@aai.ee the Milky Way high latitude (halo) regions identified several dozen young B-type stars (Saffer et al. 1997; Lindblad et al. 1997; Rolleston et al. 1999). Within $0.7 \mathrm{kpc}$ from the Sun, Hoogerwerf et al. (2000) identified 56 runaway stars. Slightly more uncertain data indicate that there are also young stars in the halo of M 31 (Hambly et al. 1995; Smoker et al. 2000).

The origin of these stars remains unclear, as well as their place of birth. Two main theories exist. According to the first theory these stars are real halo stars, i.e. they were born at high galactic latitudes as a result of collisions between high-velocity clouds (e.g. Dyson \& Hartquist 1983). However, for several stars of this kind their radial velocities indicate that they are moving away from the Galactic plane with high speed. Thus the second theory is based on the hypothesis that these stars are ejected with high velocities from the disk by some mechanism. Two main mechanisms have been considered so far - ejection via supernova explosion (Blaauw 1961), and dynamical ejection via close stellar encounters in star clusters (see e.g. Leonard \& Duncan 1988). Unfortunately, spatial velocities of these stars are poorly known and even if known they may include systematic errors (Rolleston et al. 1999).

For this reason, the study of HIP 60350 provides a good opportunity to determine the possible place of birth. In an earlier study, Maitzen et al. (1998), using simple 
dynamical considerations, estimated the time needed for the star to move from the galactic plane to its present position (about $20 \mathrm{Myrs}$ ), and speculated that the possible place of birth could be the spiral arm -II in the 4th quadrant. In the present study we use a detailed gravitational potential model of the Milky Way, integrate numerically the velocity components and coordinates backwards, and determine the place of birth in the galactic plane. We estimate also the uncertainties of the result and look for nearby star clusters.

\section{Gravitational potential of the Galaxy}

Numerical calculation of a stellar orbit presumes the knowledge of the gravitational potential of the galaxy. In the present paper, our main interest is not the long-term parameters of an orbit, i.e. the behaviour of the orbit in the far future. This kind of analysis depends essentially on the mass distribution of the Galaxy at large galactocentric distances, particularly on the distribution model of dark matter. Rather, our main aim here is to find the possible place of birth of the star. For this, it is important to know the mass distribution of the Milky Way mainly at intermediate distances. For that region, an accurate rotation curve of the Galaxy exists (construction of a mass distribution model of the Galaxy includes, beside the rotation curve, the knowledge of additional parameters, e.g. the local Oort constants, the solar distance and the circular velocity etc., see Einasto 1979 or a more recent review by Dehnen \& Binney 1998). The circular velocity is directly related to the radial derivative of the gravitational potential, which is contained in the equations of motion of a star. Several models exist which describe the gravitational potential of the Galaxy. In order to calculate the orbit of the star HIP 60350, we need to know the radial and vertical derivatives of the potential also outside the galactic plane. Hence, it is better to use models which take into account the real ellipticity of the Milky Way mass distribution, including the individual flatness of different stellar populations. For this reason we decided to use the model of the Galaxy proposed by Haud \& Einasto (1989). A recent model by Dehnen \& Binney (1998) also takes into account the real flatness of stellar populations but their algorithm for gravitational potential calculation is less convenient for our purpose.

In our model, the Galaxy is given as a superposition of different subsystems. Each subsystem represents a certain stellar/gas population with corresponding density distribution, chemical composition and kinematical characteristics. The density distribution of each component is approximated by an inhomogeneous ellipsoid of rotational symmetry with constant axial ratio $\epsilon$ (Einasto 1972; Einasto \& Haud 1989). The spatial density of visible populations is described by the law

$\rho(a)=\rho(0) \exp \left[-\left(a / a_{\mathrm{c}}\right)^{1 / N}\right]$,

where $\rho(0)=h M /\left(4 \pi \epsilon a_{0}^{3}\right)$ is the central density, $a=\sqrt{R^{2}+z^{2} / \epsilon^{2}}$ is the distance along the major axis, $a_{\mathrm{c}}=k a_{0}$ is the core radius ( $a_{0}$ is the harmonic mean radius), $h$ and $k$ are normalizing parameters, depending on the parameter $N$, which allows to vary the density behaviour with $a$. The definition of normalizing parameters and their calculation is described in Tenjes et al. (1994), Appendix B. For the disk and the flat components we use the density distribution in the following form

$\rho(a)=\rho_{+}(a)-\rho_{-}(a)$,

where subindices "+" and "_" denote density distributions (1) of components with positive and negative masses respectively. In this way, we obtain density distributions with a central density depression. If we demand that the density be zero at $a=0$ and positive elsewhere, the following relations must hold between the parameters of components $\rho_{+}$and $\rho_{-}: a_{0-}=\kappa a_{0+}, M_{-}=-\kappa^{2} M_{+}, \epsilon_{-}=\epsilon_{+} / \kappa$, where $\kappa<1$ is a parameter which determines the relative size of the hole in the centre of the disk. To avoid negative densities of the population, the structural parameters of components $N_{+}$and $N_{-}$must be equal (see Einasto et al. 1980).

The dark matter distribution is represented by a modified isothermal law

$\rho(a)= \begin{cases}\rho(0)\left(\left[1+\left(\frac{a}{a_{\mathrm{c}}}\right)^{2}\right]^{-1 / 2}-\left[1+\left(\frac{a^{0}}{a_{\mathrm{c}}}\right)^{2}\right]^{-1 / 2}\right)^{2} & a \leq a^{0} \\ 0 & a>a^{0}\end{cases}$

Here $a^{0}$ is the outer cutoff radius of the isothermal sphere.

The modeling procedure and model parameters are given in the original paper and it is not necessary to repeat them here. Knowing the density distribution formula of the components and the parameters of the populations we can calculate the gravitational potential at every point $(R, z)$.

\section{Numerical orbit calculation}

The equations of motion in cylindrical coordinates are (see e.g. Binney \& Tremaine 1987, Sect. 3.1)

$$
\begin{aligned}
L_{z} & =R v_{\psi}=\mathrm{const}, \\
\ddot{R} & =-\frac{\partial \Phi}{\partial R}+\frac{L_{z}^{2}}{R^{3}}, \\
\ddot{z} & =-\frac{\partial \Phi}{\partial z}
\end{aligned}
$$

where $\Phi$ is the gravitational potential not depending on the azimuthal coordinate $\psi$. The gravitational potential derivatives for inhomogeneous ellipsoidal mass distribution with constant ellipticity are

$$
\begin{aligned}
& \frac{\partial \Phi(R, z)}{\partial R}=R \frac{G h M}{\left(e a_{0}\right)^{3}} \int_{0}^{\arcsin (e)} \rho^{*}(a) \sin ^{2} x \mathrm{~d} x \\
& \frac{\partial \Phi(R, z)}{\partial z}=z \frac{G h M}{\left(e a_{0}\right)^{3}} \int_{0}^{\arcsin (e)} \rho^{*}(a) \tan ^{2} x \mathrm{~d} x
\end{aligned}
$$

where $a^{2}=\frac{\sin ^{2} x}{e^{2}}\left(R^{2}+\frac{z^{2}}{\cos ^{2} x}\right), e=\sqrt{1-\epsilon^{2}}$ is the eccentricity and $\rho^{*}(a)=\exp \left(-\left[a /\left(k a_{0}\right)\right]^{1 / N}\right)$. Here $\rho^{*}(a)$ is our density distribution (1). 


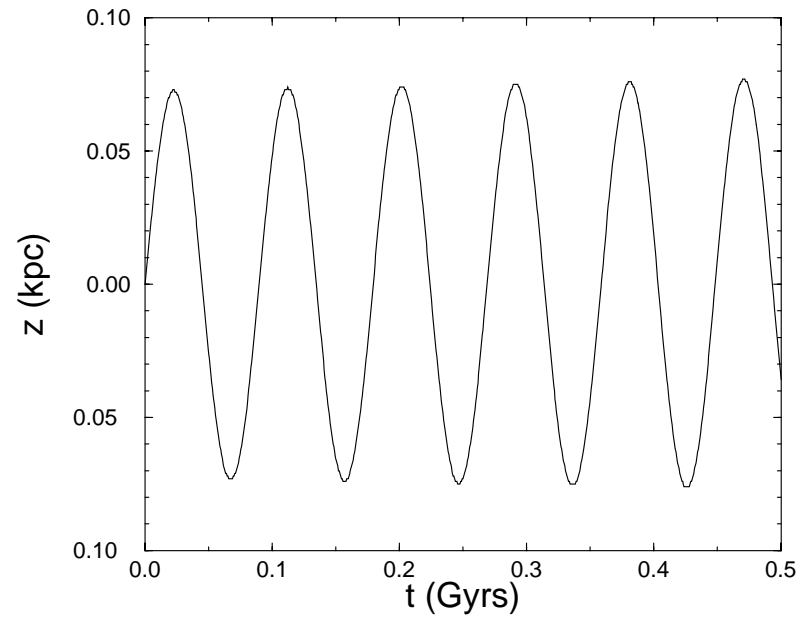

Fig. 1. Test orbit with $\left(v_{R}, v_{\psi}, v_{z}\right)=(0,217,5)$

For a spherical dark matter distribution the gravitational potential derivatives are

$$
\begin{aligned}
& \frac{\partial \Phi(R, z)}{\partial R}=R \frac{G h M}{a_{0}^{3}} \int_{0}^{1} \rho^{*}(a) u^{2} \mathrm{~d} u, \\
& \frac{\partial \Phi(R, z)}{\partial z}=z \frac{G h M}{a_{0}^{3}} \int_{0}^{1} \rho^{*}(a) u^{2} \mathrm{~d} u,
\end{aligned}
$$

where $a^{2}=u^{2}\left(R^{2}+z^{2}\right)$ and $\rho^{*}(a)$ is again the density distribution (3) without the factor $\rho(0)$.

We calculated integrals (5) and (6) numerically using the Gaussian quadrature formula with 40 points. The equations of motion (4) were solved numerically using the 4 th order Runge-Kutta method.

The correctness of the calculated orbits was tested in several ways. The most simple test is to calculate the circular velocity at some distance (e.g. at solar distance) with the known formula $V^{2}=R \partial \Phi / \partial R$, and then integrate the orbit numerically with these initial data. Doing so we found that the orbit remains circular with a precision of 0.1 percent.

Next we calculated vertical oscillations relative to the galactic plane at solar distances with $v_{z}=5 \mathrm{~km} \mathrm{~s}^{-1}$ at $z=0$. The orbit is presented in Fig. 1 . We see that the period of vertical oscillations remains constant, as it must be, and is $T=0.0896$ Gyrs. For small oscillations this period is simply related to the local mass density:

$\rho=\frac{\pi}{G T^{2}}+\frac{A^{2}-B^{2}}{2 \pi G}$.

Here $A=14.8 \mathrm{~km} \mathrm{~s}^{-1} \mathrm{kpc}^{-1}$ and $B=-12.4 \mathrm{~km} \mathrm{~s}^{-1} \mathrm{kpc}^{-1}$ are Oort's constants (Feast \& Whitelock 1997), giving the value $\rho=0.0890 M_{\odot} \mathrm{pc}^{-3}$. The direct calculation from the initial parameters of the galactic model yields as the local mass density $0.0884 M_{\odot} \mathrm{pc}^{-3}$, in very good agreement with the previous value.

Finally, we calculated a typical orbit with $\left(v_{R}, v_{\psi}\right.$, $\left.v_{z}\right)=(50,250,50)$. This orbit in the comoving meridional plane is presented in Fig. 2. We see that the orbit remains well confined within a region limited by the integrals of motion (see Kuzmin 1956; Ollongren 1962).

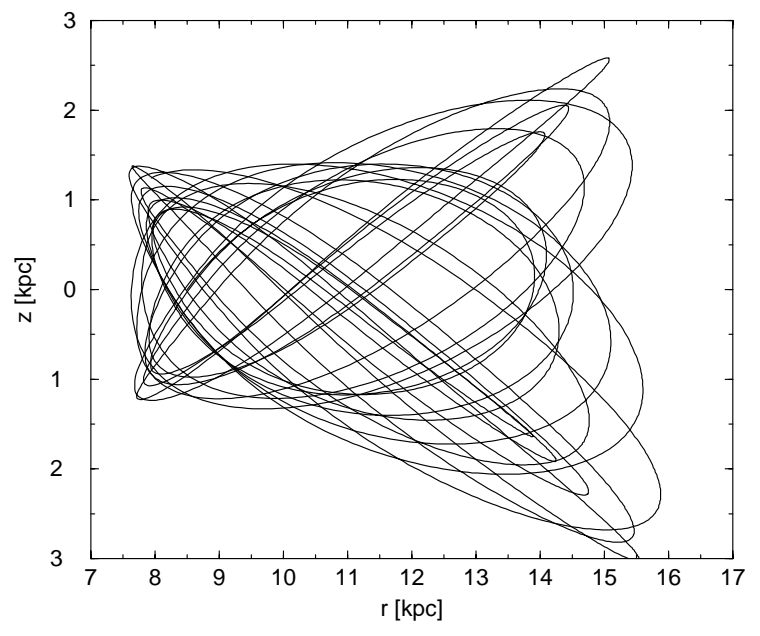

Fig. 2. A meridional section of a test orbit with $\left(v_{R}, v_{\psi}, v_{z}\right)=$ $(50,250,50)$

After these tests we began to calculate the orbit of HIP 60350.

\section{Orbit of the star HIP 60350}

Knowing the present phase space coordinates for HIP 60350 we calculated its orbit back, and found that the star was in the galactic plane 20.4 Myrs ago at $R=8.05 \mathrm{kpc}$ and $\theta=-80^{\circ}$. The spatial velocity components at that moment were $v_{r}=-278 \mathrm{~km} \mathrm{~s}^{-1}, v_{\theta}=$ $463 \mathrm{~km} \mathrm{~s}^{-1}, v_{z}=191 \mathrm{~km} \mathrm{~s}^{-1}$. Subtracting the galactic rotation component at $8 \mathrm{kpc} v_{\theta}^{0}=220 \mathrm{~km} \mathrm{~s}^{-1}$ we obtain that the ejection velocity was $v=416 \mathrm{~km} \mathrm{~s}^{-1}$. The projection of the orbit into the plane of the Galaxy is shown in Fig. 3 by a bold solid line.

In order to examine possible ejection mechanisms it is needed to estimate the uncertainties in our result. First, we estimate errors due to the galactic mass model. An acceptable model must be in accordance with measured galactic rotation velocities. Thus, the mass of the galactic disk (which is the most essential parameter in our case) must give rotation velocities within the measured velocity errors. The rotation curve at distances $R \sim 8-10 \mathrm{kpc}$ from the Galactic centre is known with errors $\pm 10 \mathrm{~km} \mathrm{~s}^{-1}$ (Fish \& Tremaine 1990; Binney \& Merrifield 1998). These errors allow us to vary the disk mass within the limits $\pm 0.910^{10} M_{\odot}$. Orbit calculations for galactic models with disk masses $5.910^{10} M_{\odot}$ and $7.810^{10} M_{\odot}$ show that the position of galactic plane crossing has an uncertainty of only $\pm 0.1 \mathrm{kpc}$, which is rather small.

Second, we studied uncertainties due to the errors in the observed velocity components of the star, quoted by Maitzen et al. (1998) to be 15 percent. To the $\theta$ component velocity we must add the circular velocity of LSR $\left(220 \pm 10 \mathrm{~km} \mathrm{~s}^{-1}\right)$. Thus the uncertainties of the velocity components are $( \pm 50, \pm 30, \pm 20)$. To estimate the influence of velocity uncertainties of each component on the position of the star at the galactic plane crossing, we calculated orbits of the star for a number of observed 
velocities including estimated errors. We find that the resulting overall uncertainty of the plane crossing lies approximately within an ellipse with semiaxes $1.1 \mathrm{kpc}$ and $0.7 \mathrm{kpc}$. The long axis of the ellipse is oriented approximately in the direction of the stellar orbit (Fig. 3, dashed ellipse). Hence we must search for the possible birthplace of HIP 60350 within this region.

Because our star is young, we are interested in the presence of young star clusters and associated interstellar gas regions. According to a catalog of HII regions of the Milky Way by Georgelin \& Georgelin (1976), potential candidates could be objects Nos. 56, 57, 60 and 62 . In addition, we looked for promising candidates in the molecular gas survey by Grabelsky et al. (1988); clouds Nos. 19, 22 and 27 are candidates. From these lists only clouds Nos. 60 and 62 are known to contain stars at present. Moreover, cloud No. 62 corresponds to the well-known star cluster NGC 3603; thus, the distance to this cluster is known with high accuracy. The distances to other clouds without known associated stars are estimated by the authors of corresponding papers using indirect data and are less certain (distances to the clouds Nos. 56 and 57 are estimated by Georgelin \& Georgelin 1976 on the basis of galactic northern and southern rotation models, distances to the clouds Nos. 19, 22 and 27 are estimated by Grabelsky et al. 1988 on the basis of radius-line-width relation; in both papers the authors used the old values of galactic constants $\left(10 \mathrm{kpc}\right.$ and $\left.250 \mathrm{~km} \mathrm{~s}^{-1}\right)$, thus we transformed the clouds distances to the new values of galactic constants recommended by the IAU, see Kerr \& LyndenBell 1986). As the star HIP 60350 started from the galactic plane about $20 \mathrm{Myr}$ ago we must transfer the positions of the selected clouds back by that time assuming circular orbits at their galactocentric distances. The results are presented in Fig. 3.

The positions of young objects in the galactic plane may be influenced by their peculiar velocity component in the $R$-direction. Peculiar velocities of young star clusters are typically $\leq 15 \mathrm{~km} \mathrm{~s}^{-1}$ (see e.g. Nezhinskij et al. 1995), giving during a $20 \mathrm{Myr}$ orbit a maximum correction of the position up to $\pm 0.3 \mathrm{kpc}$. Thus we see that clouds Nos. 60 and 62 , which are known to contain stars, as well as molecular clouds, may lie inside the boundary of the permitted region of the birthplace of our star.

Recent observations (Brandl et al. 1999; De Pree et al. 1999) allowed us to study in detail the star cluster NGC 3603. According to these observations this cluster lies at a distance $6.1 \pm 0.6 \mathrm{kpc}$ from the Sun. NGC 3603 contains a lot of young and massive stars and is still in the stage of star formation. Its position corresponds to the birthplace of HIP 60350 nearly perfectly. Related to the cluster HII region is one of the largest HII regions in the Galaxy. The initial mass function (IMF) of the cluster extends up to $120 M_{\odot}$ (Drissen et al. 1995).

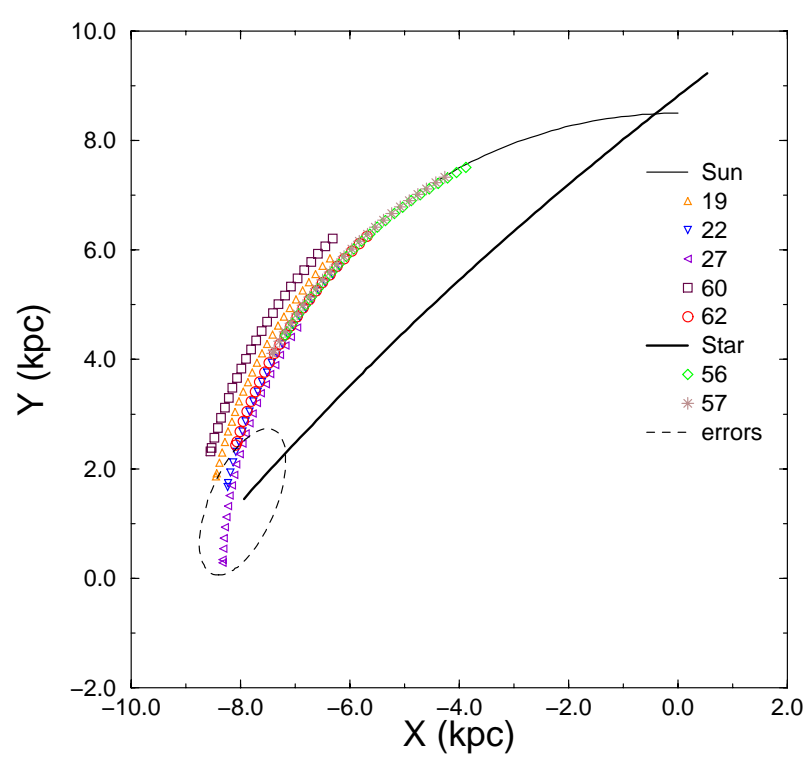

Fig. 3. Motion of clouds Georgelin 56, 57, 60 and 62 (= NGC 3603), Grabelsky 19, 22, and 27, the Sun and the star HIP 60350 at the Galactic plane during 20.4 Myrs. For HIP 60350 the orbit projected to the Galactic plane is given

\section{Discussion}

In the present work we integrated the orbit of the extreme runaway star HIP 60350 back to the galactic plane. Taking into account possible errors in the velocity measurements and the galactic model we have found the probable birthplace of this star.

OB runaway stars can be produced either by supernova explosions in massive close binaries or by close dynamical encounters in dense star clusters. First we discuss the ejection as a result of SN explosion. This mechanism was studied first by Blaauw (1961) as a symmetric SN explosion where the momentum of ejected matter was balanced by the momentum of the binary centre-of-mass. However, before the SN explosion there must occur some mass transfer between the system components, and thus the amount of ejected matter is less than half of the system's total mass. Disruption of a binary system is unlikely and the maximum velocity of the centre-of-mass cannot be too large. According to Tauris \& Bailes (1996), the limit for the recoil velocity is $270 \mathrm{~km} \mathrm{~s}^{-1}$ (see also Nelemans et al. 1999).

According to recent studies, supernova explosions can be asymmetric. To explain the observed spatial velocity distribution of single pulsars, we need to assume asymmetric SN explosions, where the newborn neutron star, due to an additional kick, attains a mean velocity of $450 \mathrm{~km} \mathrm{~s}^{-1}$ in an arbitrary direction (Lyne \& Lorimer 1994; Hartmann 1997). This explosion probably disrupts the system, and thus runaway stars should not be presently binary stars. Although an asymmetric explosion may give a very high velocity to the pulsar (up to $1500 \mathrm{~km} \mathrm{~s}^{-1}$ ), its impact to the companion star is significantly smaller. According to calculations by Tauris \& Takens (1998) the spatial velocity of the companion star depends on several parameters (kick speed, companion star mass etc.); usually it does not 
exceed $300 \mathrm{~km} \mathrm{~s}^{-1}$. Applying these results to our $5 M_{\odot}$ star, the runaway velocity is less than $200 \mathrm{~km} \mathrm{~s}^{-1}$, even for the largest kick speeds.

Because it is difficult to obtain the ejection velocity of $420 \mathrm{~km} \mathrm{~s}^{-1}$ with a SNe scenario, we turn our attention to dynamical interactions. Dynamical interactions have been studied by Leonard \& Duncan (1988), who showed that typical velocities in interactions extend up to $250 \mathrm{~km} \mathrm{~s}^{-1}$. According to models the most efficient in producing runaway stars are binary-binary interactions (Mikkola 1983). The efficiency of binary-binary interactions dominates over binary-single interactions, especially in producing high-velocity runaway stars (Leonard \& Duncan 1988). However, these are the results of N-body experiments and were limited by available computational time. For this reason Leonard (1991) performed special numerical experiments with binary-binary interactions to determine the maximum possible velocity of ejection. His results were presented for different ratios $v_{\mathrm{ej}} / v_{\mathrm{esc}}\left(v_{\mathrm{ej}}-\right.$ ejection velocity of a star, $v_{\text {esc }}$ - escape velocity at the surface of ejected star).

HIP 60350 was ejected from the galactic plane with a spatial velocity of $420 \mathrm{~km} \mathrm{~s}^{-1}$. A $5 M_{\odot}$ star has a radius of $2.6 R_{\odot}$ (Tout et al. 1996) and $v_{\text {esc }}=840 \mathrm{~km} \mathrm{~s}^{-1}$, thus $v_{\mathrm{ej}}=0.50 v_{\mathrm{esc}}$. According to Leonard (1991, his Table 3) the ejection velocity $v_{\text {ej }}=0.50 v_{\text {esc }}$ cannot be obtained if HIP 60350 interacted with equal or lower mass stars. Such high ejection velocities can be obtained by a star in interactions with three stars having masses at least 4 times larger, but probably even 8 times larger. In this case sufficient ejection velocities for the least massive companion may appear within about 400 cluster crossing times. Hence to produce a high-velocity star like HIP 60350 , the participation of 3 stars with masses $\sim 40 M_{\odot}$ is needed. Typical diameters of young open clusters are 1-9 pc (Phelps \& Janes 1993), the line-of-sight velocity dispersion is about $3-4 \mathrm{~km} \mathrm{~s}^{-1}$ (see e.g. Kroupa 2000), thus the resulting crossing time is $0.3-2 \mathrm{Myr}$. On the other hand, according to photometric measurements, HIP 60350 is clearly rather close to the ZAMS, and cannot be old (Maitzen et al. 1998). It is very plausible that the ejecting event took place in an early phase of star formation in a very compact cluster, where IMF was skewed towards the high-mass end compared with the field star IMF (Clarke \& Pringle 1992). If this is the case, stars of larger mass could indeed participate in the ejection event (MS lifetimes of $20 M_{\odot}$ and $40 M_{\odot}$ stars are $5 \mathrm{Myr}$ and $2 \mathrm{Myr}$, respectively).

Dynamical interactions are more efficient in massive and compact clusters (Leonard \& Duncan 1990). Interactions (ejections, mergers) are also more frequent in clusters with active star formation at early evolutionary stages (Bonnell et al. 1998; Portegies Zwart et al. 1999). For this reason the cluster G $62=$ NGC 3603 seems to be a suitable candidate. The IMF of the cluster extends up to large masses, making high ejection velocities reasonable. When varying the observed velocity of HIP 60350 (but remaining within observational errors) we calculated that with the present velocity components $v_{\mathrm{r}}=318 \mathrm{~km} \mathrm{~s}^{-1}$,

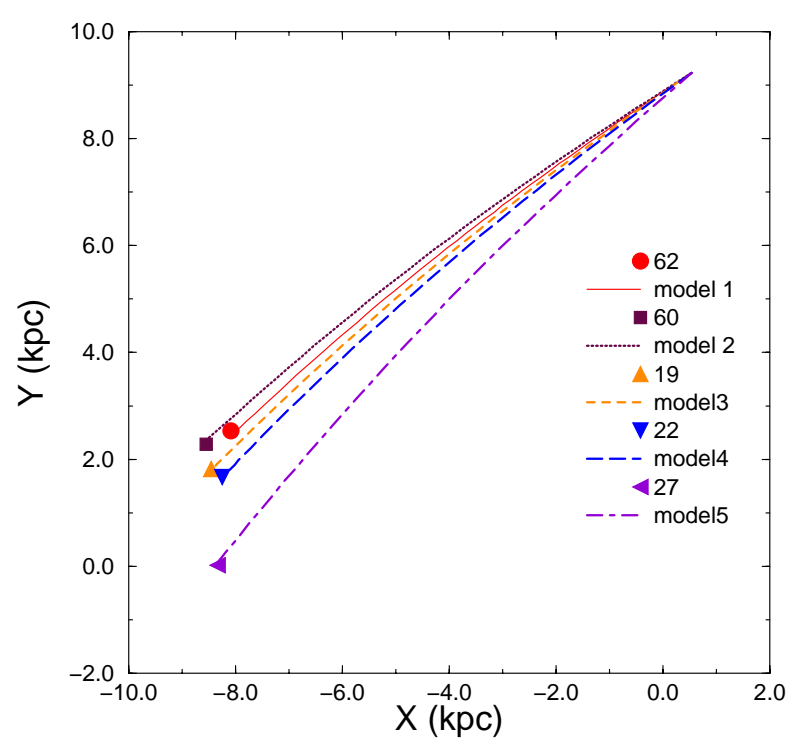

Fig. 4. Positions of clouds at the times of star ejection. Corresponding orbits of the ejected star are labeled as different models with parameters given in Table 1

Table 1. Parameters of stellar orbits for individual clouds

\begin{tabular}{lllllll}
\hline \multirow{2}{*}{ Model } & \multicolumn{3}{c}{ Present velocity $\left(\mathrm{km} \mathrm{s}^{-1}\right)$} & $\begin{array}{l}\text { Ejection } \\
\text { time }(\mathrm{Myr})\end{array}$ & $\begin{array}{l}\text { Ejection } \\
\text { velocity }\end{array}$ \\
\cline { 2 - 5 } & $v_{\mathrm{r}}$ & $v_{\theta}$ & $v_{\mathrm{z}}$ & 19.9 & 405 \\
\hline 1 & 318 & 423 & 138 & 20.7 & 402 \\
2 & 307 & 428 & 130 & 20.7 & 416 \\
3 & 332 & 423 & 130 & 20.5 & 420 \\
4 & 343 & 418 & 130 & 21.6 & 456 \\
5 & 397 & 403 & 117 & & \\
\hline
\end{tabular}

$v_{\theta}=423 \mathrm{~km} \mathrm{~s}^{-1}$ and $v_{\mathrm{z}}=138 \mathrm{~km} \mathrm{~s}^{-1}$ the place of birth of the star coincides with the position of G62 (Fig. 4, circle and continuous line, see also Table 1, Model 1).

However, there exists a serious argument against NGC 3603 as the birthplace of HIP 60350. The age of NGC 3603 is estimated to be only 3-4 Myrs (De Pree et al. 1999), which is in contradiction to an ejection 20 Myrs ago. Further, some time is also needed for interactions - statistically up to $400 t_{\text {cross }} \geq 100 \mathrm{Myr}$. This discrepancy may not be critical, because star formation in young clusters is a complicated process with different stages (see e.g. Elmegreen 2000 and for NGC 3603, Brandner et al. 1997). Thus, we would like not to exclude this cluster together with its surrounding region as a candidate for the origin of HIP 60350. The age structure of the cluster deserves special and careful study: Eisenhauer et al. (1998) derived that the distribution of stellar ages in NGC 3603 is non-Gaussian and extends up to $100 \mathrm{Myr}$.

Another candidate is the HII region G60, which is also situated quite near to the probable ejection place. With present velocity components $v_{\mathrm{r}}=307 \mathrm{~km} \mathrm{~s}^{-1}, v_{\theta}=$ $428 \mathrm{~km} \mathrm{~s}^{-1}$ and $v_{\mathrm{z}}=130 \mathrm{~km} \mathrm{~s}^{-1}$ the ejection place will lie close to G60 (Fig. 4, Table 1, model 2). The HII region 
G60 is less studied. We do not know the detailed structure and age of this region. These properties remain suitable for further study.

According to the study of molecular clouds by Grabelsky et al. (1988), near the birthplace of HIP 60350 there are clouds of molecular gas Nos. 19, 22 and 27 according to his designations (in addition cloud Nos. 17 corresponds to NGC 3603). For these clouds the corresponding models are $3-5$, see Table 1 . Unfortunately the distances to these clouds are kinematic (they are based on empirical radius-line-width relations by Dame et al. 1986, see Grabelsky et al.) and are thus uncertain. Moreover, these clumps may be blended together at lower intensities, and SN explosions (Öpik 1954) and stellar winds from massive stars (Carpenter et al. 1995) may disrupt the original cloud structure. Molecular clouds have lifetimes of 10-100 Myr (Blitz \& Williams 2000; Williams et al. 2000) or less (Ballesteros-Paredes et al. 1999; Elmegreen 2000 ), which is probably too short to produce high ejection velocities. Thus, it is not likely that the referred molecular clouds can be directly assigned as birthplaces of HIP 60350.

At present the place of birth of our star is at $l=298$, $b=0$ and $R=6.9 \mathrm{kpc}$ from the Sun. This region (see also the ellipse of errors in Fig. 3) deserves further careful study. According to our analysis it is probable that in this region there is an open cluster with an age of more than 20 Myr. Finding this kind of star cluster is not simple, due to the relatively large distance and possible ISM obscuring (unless we are lucky enough to find it in a cloud hole). In order to decrease the probable search area from where the ejection took place it seems most promising to determine more precise velocity measurements for the star.

Individually identified molecular clouds referred to above may simply be remnants of an earlier star formation process. Together with NGC 3603 they may form a bigger stellar-gaseous complex. Observations by Grabelsky et al. (1988) hint that near NGC 3603 a more massive and extremely disrupted cloud complex exists which refers to past intensive star formation. Massive stars from this first star formation stage ejected HIP 60350, exploded as supernovae and triggered star formation in the adjecent parts of the original cloud. These events might be similar to the scenario outlined by Preibisch \& Zinnecker (1999) for Scorpius-Centaurus OB association.

Observations of different individual OB runaways support both the supernova explosion scenario (e.g. Kaper et al. 1997) and the cluster ejection model (e.g. Ryans et al. 1999; Moffat et al. 1998); both scenarios produce runaways (see recent detailed analysis about nearby runaways by Hoogerwerf et al. 2000). The very high velocity runaway HIP 60350 seems to have been ejected via dynamical ejection from G62 or from G60.

Finally, we have calculated the future orbit of the star HIP 60350, starting from its present position. The total galactocentric velocity of the star is less than the escape velocity at $R=9 \mathrm{kpc}, 600 \mathrm{~km} \mathrm{~s}^{-1}$, thus it must remain within the potential well of the Galaxy. The projection

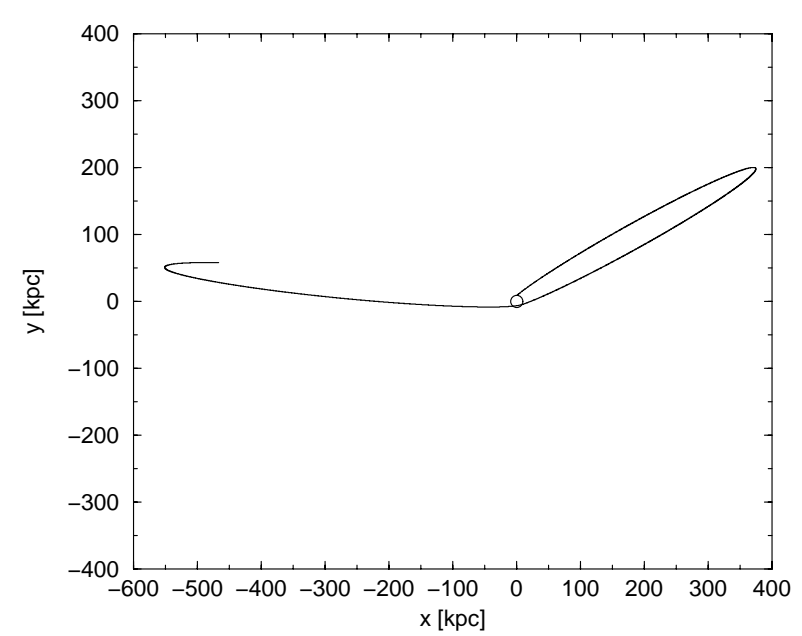

Fig. 5. The orbit of the star HIP 60350 for the next 13.5 Gyrs, projected to the galactic plane. Circle with centre coordinates $(0,0)$ is situated at the galactic centre and has a radius $10 \mathrm{kpc}$. The $x$ and $y$ axes are directed toward the direction of rotation (at Sun position) and away from the centre toward the Sun, respectively

of the orbit to the galactic plane is shown in Fig. 5. We see that its apogalactic distance reaches in the first and second revolution 425 and $554 \mathrm{kpc}$ at 3.3 and 11.4 Gyrs, respectively. Evidently, at these large distances, perturbations by other members of the Local Group galaxies are important. Most likely the star will remain in the common potential well of the whole Local Group.

Acknowledgements. We would like to thank the anonymous referee for useful comments and suggestions. PT acknowledges financial support from the Estonian Science Foundation (grant 2627); JE acknowledges financial support from the Estonian Science Foundation (grant 2625) and hospitality during visits to Institut für Astronomie der Universität Wien.

\section{References}

Ballesteros-Paredes, J., Hartmann, L., \& Vázquez-Semanini, E. 1999, ApJ, 527, 285

Binney, J., \& Merrifield, M. 1998, Galactic astronomy (Princeton University Press, Princeton, New Jersey)

Binney, J., \& Tremaine, S. 1987, Galactic dynamics (Princeton University Press, Princeton, New Jersey)

Blaauw, A. 1961, BAN, 15, 265

Blitz, L., \& Williams, J. P. 2000. Molecular clouds, in The physics of star formation and early stellar evolution, ed. N. Kylafis, \& C. J. Lada, in press [astro-ph/9903382]

Bonnell, I. A., Bate, M. R., \& Zinnecker, H. 1998, MNRAS, 298, 93

Brandl, B., Brandner, W., Grebel, E. K., \& Zinnecker, H. 1999, The Messenger, Nr, 98, 46

Brandner, W., Grebel, E. K., Chu, Y.-H., \& Weiss, K. 1997, ApJ, 475, L45

Carpenter, J. M., Snell, R. L., \& Schloerb, F. P. 1995, ApJ, 450, 201

Clarke, C. J., \& Pringle, J. E. 1992, MNRAS, 255, 423

Dame, T. M., Cohen, R. S., Elmegreen, B. G., \& Thaddeus, P. 1986, ApJ, 305, 892 
Dehnen, W., \& Binney, J. 1998, MNRAS, 294, 429

De Pree, C. G., Nysewander, M. C., \& Goss, W. M. 1999, AJ, 117, 2902

Drissen, L., Moffat, A. F. J., Walborn, N. R., \& Shara, M. M. 1995, AJ, 110, 2235

Dyson, J. E., \& Hartquist, T. W. 1983, MNRAS, 203, 1233

Einasto, J. 1972. Tartu Astr. Obs. Teated, 40, 3, also in Stars and the Milky Way System, Proc. First European Ast. Meet., vol. 2, ed. L. N. Mavridis (Springer, Berlin, Heidelberg, New York), 291

Einasto, J. 1979, Galactic mass modeling, in The large-scale characteristics of the Galaxy, ed. W. B. Burton (Kluwer, Dordrecht), 451

Einasto, J., \& Haud, U. 1989, A\&A, 223, 89

Einasto, J., Tenjes, P., Barabanov, A. V., \& Zasov, A. V. 1980, Ap\&SS, 67, 31

Eisenhauer, F., Quirrenbach, A., Zinnecker, H., \& Grenzel, R. 1998, ApJ, 498, 278

Elmegreen, B. G. 2000, ApJ, 530, 277

Feast, M., \& Whitelock, P. 1997, MNRAS, 291, 683

Fish, M., \& Tremaine, S. 1990, ARA\&A, 29, 409

Georgelin, Y. M., \& Georgelin, Y. P. 1976, A\&A, 49, 57

Grabelsky, D. A., Cohen, R. S., Bronfman, L., \& Thaddeus, P. 1988, ApJ, 331, 181

Hambly, N. C., Fitzsimmons, A., Keenan, F. P., et al. 1995, ApJ, 448, 628

Hartman, J. W. 1997, A\&A, 322, 127

Haud, U., \& Einasto, J. 1989, A\&A, 223, 59

Hoogerwerf, R., de Bruijne, J. H. J., \& de Zeeuw, P. T. 2000, A\&A, in press [astro-ph/0010057]

Kaper, L., van Loon, J. Th., Augusteijn, T., et al. 1997, ApJL, 475, L37

Kerr, F. J., \& Lynden-Bell, D. 1986, MNRAS, 221, 1023

Kroupa, P. 2000, New Astron., 4, 615

Kuzmin, G. 1956, Astr. Zh., 33, 27

Leonard, P. J. T. 1991, AJ, 101, 562

Leonard, P. J. T., \& Duncan, M. J. 1988, AJ, 96, 222

Leonard, P. J. T., \& Duncan, M. J. 1990, AJ, 99, 608

Lindblad, P. O., Lodén, K., Palouš, J., \& Lindegren, L. 1997,
Runaway stars and the force perpendicular to the galactic plane. in Hipparcos - Venice '97, ESA, p. 665 (=//astro.estec.esa.nl/Hipparchos/venice.html)

Lyne, A. G., \& Lorimer, D. R. 1994, Nature, 369, 127

Maitzen, H. M., Paunzen, E., Pressberger, R., Slettebak, A., \& Wagner, R. M. 1998, A\&A, 339, 782

Mikkola, S. 1983, MNRAS, 203, 1107

Moffat, A. F. J., Marchenko, S. V., Seggewiss, W., et al. 1998, A\&A, 331, 949

Nelemans, G., Tauris, T. M., \& van den Heuvel, E. P. J. 1999, A\&A, 352, L87

Nezhinskij, E. M., Ossipkov, L. P., \& Kutuzov, S. A. 1995, Open cluster systems: Kinematics, orbits, in Stellar populations, ed. P. C. van der Kruit, \& G. Gilmore (Kluwer, Dordrecht), 374

Ollongren, A. 1962, BAN, 16, 241

Öpik, E. 1954, Irish AJ, 2, 219

Phelps, R. L., \& Janes, K. A. 1993, AJ, 106, 1870

Portegies Zwart, S. F., Makino, J., McMillan, S. L. W., \& Hut, P. 1999, A\&A, 348, 117

Preibisch, T., \& Zinnecker, H. 1999, AJ, 117, 2381

Rolleston, W. R. J., Hambly, N. C., Keenan, F. P., Dufton, P. L., \& Saffer, R. A. 1999, A\&A, 347, 69

Ryans, R. S. I., Keenan, F. P., Rolleston, W. R. J., Sembach, K. R., \& Davies, R. D. 1999, MNRAS, 304, 947

Saffer, R. A., Keenan, F. P., Hambly, N. C., Dufton, P. L., \& Liebert, J. 1997, ApJ, 491, 172

Smoker, J. V., Keenan, F. P., Marcha, M. J., Watson, D., \& Irwin, M. J. 2000, A\&A, 361, 60

Tauris, T. M., \& Bailes, M. 1996, A\&A, 315, 432

Tauris, T. M., \& Takens, R. J. 1998, A\&A, 330, 1047

Tenjes, P., Haud, U., \& Einasto, J. 1994, A\&A, 286, 753

Tout, C. A., Pols, O. R., Eggleton, P. P., \& Han, Z. 1996, MNRAS, 281, 257

Williams, J. P., Blitz, L., \& McKee, C. F. 2000, The structure and evolution of molecular clouds, in Protostars and planets IV. Tucson, ed. V. Mannings, \& A. Boss (Univ. Arizona Press) 